\title{
Light front wave functions and diffractive electroproduction of vector mesons
}

\author{
Chao Shi॰, ${ }^{1, *}$ Ya-Ping Xie, ${ }^{2, \dagger}$ Ming Li, ${ }^{1}$ Xurong Chen $\odot,{ }^{2,3}$ and Hong-Shi Zong ${ }^{4}$ \\ ${ }^{1}$ Department of Nuclear Science and Technology, Nanjing University of Aeronautics and Astronautics, \\ Nanjing 210016, China \\ ${ }^{2}$ Institute of Modern Physics, Chinese Academy of Sciences, Lanzhou 730000, China \\ ${ }^{3}$ Guangdong Provincial Key Laboratory of Nuclear Science, Institute of Quantum Matter, \\ South China Normal University, Guangzhou 510006, China \\ ${ }^{4}$ Department of Physics, Nanjing University, Nanjing 210093, China
}

(Received 2 February 2021; accepted 8 October 2021; published 3 November 2021)

\begin{abstract}
We determine the leading Fock-state light front wave functions (LF-LFWFs) of the $\rho$ and $J / \psi$ mesons, for the first time from the Dyson-Schwinger and Bethe-Salpeter equations approach. A unique advantage of this method is that it renders a direct extraction of LF-LFWFs in presence of a number of higher Fockstates. Modulated by the current quark mass and driven by the dynamical chiral symmetry breaking, we find the $\rho$ and $J / \psi$ LF-LFWFs different in profile, i.e., the former are broadly distributed in $x$ (the longitudinal light-cone momentum fraction of meson carried by quark) while the latter are narrow. Moreover, the $\rho$ LF-LFWFs contribute less than $50 \%$ to the total Fock-state normalization, suggesting considerable higher Fock-states in $\rho$. We then use these LF-LFWFs to study the diffractive $\rho$ and $J / \psi$ electroproduction within the dipole picture. The calculated cross section shows general agreement with HERA data, except for growing discrepancy in $\rho$ production at low photon virtuality.
\end{abstract}

DOI: 10.1103/PhysRevD.104.L091902

\section{INTRODUCTION}

Diffractive vector meson production provides an important probe to the gluon saturation [1]. Within the dipole picture, saturation and non-saturation scattering amplitudes yield sizable effects in, e.g., the t-distribution of differential cross section [2], the cross section ratio between $\mathrm{eAu} \rightarrow \mathrm{eAuV}$ and ep $\rightarrow$ epV [3]. Meanwhile, the LF-LFWFs of vector mesons and photon are important nonperturbative element of the dipole picture. Their determination in connection with QCD greatly helps reduce the theoretical uncertainties, and substantially deepens our understanding of the hard diffractions.

While the non-relativistic QCD (NRQCD) sheds light on heavy meson LFWFs $[1,4,5]$, it remains a great challenge to calculate light vector meson LFWFs in connection with QCD to date. The light-cone QCD Hamiltonian, which encodes abundant creation and annihilation of light-quarks and gluons, gets intensely difficult to diagonalize with increasing number of Fock-states [6]. Therefore, existing dipole picture

\footnotetext{
*cshi@nuaa.edu.cn

xieyaping@impcas.ac.cn
}

Published by the American Physical Society under the terms of the Creative Commons Attribution 4.0 International license. Further distribution of this work must maintain attribution to the author(s) and the published article's title, journal citation, and DOI. Funded by SCOAP. studies all employed phenomenological (or effective) $\rho$ LFLFWFs that take the leading Fock-state truncation [7-10], i.e., the $\rho$ meson contains only the $|q \bar{q}\rangle$ component. However, in a light system as $\rho$, the quantum fluctuation can be active, so the higher Fock-states can be potentially considerable.

In this work, we introduce a novel approach based on Dyson-Schwinger and Bethe-Salpeter equations (DS-BSEs) study [11-13]. The modern DS-BSEs study is closely connected with QCD, i.e., it incorporates the quark and gluon degrees of freedom and selectively re-sums infinitely many Feynman diagrams while respecting various symmetries of QCD $[14,15]$. We then project the $\rho$ and $J / \psi$ covariant BS wave functions onto the light front and extract the LF-LFWFs from the many Fock-states embedded [16-18]. We then confront these LF-LFWFs with diffractive $\rho$ and $J / \psi$ electroproduction at hadron-electron ring accelerator (HERA) within the color dipole approach.

\section{LF-LFWFs OF $\rho$ AND $J / \psi$}

The leading Fock-state configuration of a vector meson state is expressed with the nonperturbative LFWFs $\Phi_{\lambda, \lambda^{\prime}}^{\Lambda}$

$$
\begin{aligned}
|M\rangle^{\Lambda}= & \sum_{\lambda, \lambda^{\prime}} \int \frac{d^{2} \boldsymbol{k}_{T}}{(2 \pi)^{3}} \frac{d x}{2 \sqrt{x \bar{x}}} \frac{\delta_{i j}}{\sqrt{3}} \\
& \times \Phi_{\lambda, \lambda^{\prime}}^{\Lambda}\left(x, \boldsymbol{k}_{T}\right) b_{f, \lambda, i}^{\dagger}\left(x, \boldsymbol{k}_{T}\right) d_{f, \lambda^{\prime}, j}^{\dagger}\left(\bar{x}, \overline{\boldsymbol{k}}_{T}\right)|0\rangle .
\end{aligned}
$$


The $\boldsymbol{k}_{T}=\left(k^{x}, k^{y}\right)$ is the transverse momentum of the quark $f$, and $\overline{\boldsymbol{k}}_{T}=-\boldsymbol{k}_{T}$ for antiquark $\bar{f}$. The longitudinal momentum fraction carried by quark is $x=\frac{k^{+}}{P^{+}}$, and $\bar{x}=1-x$ for antiquark. The $i$ and $j$ are color indices. The quark helicity $\lambda$ runs through $\uparrow$ and $\downarrow$, while the meson helicity $\Lambda$ runs through 0 and \pm 1 . The $\Phi\left(x, \boldsymbol{k}_{T}\right)$ 's can be further expressed with amplitudes $\psi\left(x, \boldsymbol{k}_{T}^{2}\right)$ 's which contain only scalars arguments $x$ and $\boldsymbol{k}_{T}^{2}$ [19]. Denoting the quark helicity $\uparrow=+$ and $\downarrow=-$, and omitting the function arguments, one finds for longitudinally polarized mesons

$$
\Phi_{ \pm, \mp}^{0}=\psi_{(1)}^{0}, \quad \Phi_{ \pm, \pm}^{0}= \pm k_{T}^{(\mp)} \psi_{(2)}^{0},
$$

with $k_{T}^{( \pm)}=k^{x} \pm i k^{y}$, and for transverse mesons $(\Lambda= \pm 1)$

$$
\begin{array}{ll}
\Phi_{ \pm, \pm}^{ \pm 1}=\psi_{(1)}^{1}, & \Phi_{ \pm, \mp}^{ \pm 1}= \pm k_{T}^{( \pm)} \psi_{(2)}^{1}, \\
\Phi_{\mp, \pm}^{ \pm 1}= \pm k_{T}^{( \pm)} \psi_{(3)}^{1}, & \Phi_{\mp, \mp}^{ \pm 1}=\left(k_{T}^{( \pm)}\right)^{2} \psi_{(4)}^{1} .
\end{array}
$$

Note the $\Lambda=-1$ meson can be obtained from $\Lambda=+1$ with a $\hat{Y}$ transform, which consists a parity operation followed by a $180^{\circ}$ rotation around the y axis [19]. With the help of $\hat{Y}$ and charge parity, we find the constraints

$$
\psi_{(i)}^{\Lambda}\left(x, \boldsymbol{k}_{T}^{2}\right)=\psi_{(i)}^{\Lambda}\left(1-x, \boldsymbol{k}_{T}^{2}\right),
$$

with one exception

$$
\psi_{(2)}^{1}\left(x, \boldsymbol{k}_{T}^{2}\right)=-\psi_{(3)}^{1}\left(1-x, \boldsymbol{k}_{T}^{2}\right) .
$$

In the end, for $\rho^{0}$ (with isospin symmetry) or $J / \psi$ that has charge parity, there are totally five independent $\psi_{(i)}^{\Lambda}$ 's at leading Fock state.

In this work, we derive the relation between the vector meson LF-LFWFs and its BS wave function $\chi_{\mu}^{M}(k, P)$ as

$$
\begin{aligned}
\Phi_{\lambda, \lambda^{\prime}}^{\Lambda}\left(x, \boldsymbol{k}_{T}\right)= & -\frac{1}{2 \sqrt{3}} \int \frac{d k^{-} d k^{+}}{2 \pi} \delta\left(x P^{+}-k^{+}\right) \\
& \times \operatorname{Tr}\left[\Gamma_{\lambda, \lambda^{\prime} \gamma^{+}} \chi^{M}(k, P) \cdot \epsilon_{\Lambda}(P)\right],
\end{aligned}
$$

by generalizing the projection method for pseudoscalar meson in Refs. [12,13,20]. The $\epsilon_{\Lambda}(P)$ is the meson polarization vector. The $\Gamma_{ \pm, \mp}=I \pm \gamma_{5}$ and $\Gamma_{ \pm, \pm}=$ $\mp\left(\gamma^{1} \mp i \gamma^{2}\right)$ projects out certain (anti)quark helicity configuration. The $\chi_{\mu}^{M}(k, P)$ can be expressed with the dressed quark propagator $S(k)$ and BS amplitude $\Gamma_{\mu}^{M}(k, P)$ as $\chi_{\mu}^{M}(k, P)=S(k+P / 2) \Gamma_{\mu}^{M}(k, P) S(k-P / 2)$. The trace is taken over Dirac, color, and flavor indices. An implicit color factor $\delta_{i j}$ is associated with $\Gamma_{\lambda, \lambda^{\prime}}$, as well as a flavor factor $\operatorname{diag}(1 / \sqrt{2},-1 / \sqrt{2})$ for $\rho^{0}$.

Within the DS-BSEs framework, the $S(k)$ and $\Gamma_{\mu}(k, P)$ can be solved by aligning the quark's DSE and meson's
BSE. In practice, truncations and modeling are indispensable. A longstanding and successful realization is the rainbow-ladder (RL) truncation combined with the MarisTandy model [21]. With only two model parameters, it well describes a range of hadron properties, including the pion and $\rho$ meson masses, decay constants and various elastic and transition form factors [21-26]. The success also extends to nucleon by solving the Faddeev equation $[27,28]$. A nice property of the RL truncation is that it preserves the (near) chiral symmetry of QCD by respecting the axial vector Ward-Takahashi identity [22], so it is capable of describing simultaneously the almost massless pion as a Goldstone boson and the much more massive $\rho$ and nucleon, reflecting different aspects of the dynamical chiral symmetry breaking (DCSB). Here we will explore the prediction of RL DS-BSEs on the vector meson LF-LFWFs.

The RL truncation starts with the rainbow truncation $[29,30]$

$$
Z_{1} g^{2} D_{\mu \nu}(k) \Gamma_{\nu}(k, p) \rightarrow Z_{2}^{2} k^{2} \mathcal{G}\left(k^{2}\right) D_{\mu \nu}^{\mathrm{free}}(k) \gamma_{\nu},
$$

where $Z_{1}$ and $Z_{2}$ are the renormalization constants of quark-gluon vertex and quark field respectively. The strong coupling constant $g$, as well as the dressing effects in full gluon propagator $D_{\mu \nu}(k)$ and quark-gluon vertex $\Gamma_{\nu}(k, p)$, are absorbed into a modeling function $\mathcal{G}\left(k^{2}\right)$. Here we employ the Qin-Chang (QC) model [31]

$\mathcal{G}(s)=\frac{8 \pi^{2}}{\omega^{4}} D \mathrm{e}^{-s / \omega^{2}}+\frac{8 \pi^{2} \gamma_{m}}{\ln \left[\tau+\left(1+s / \Lambda_{\mathrm{QCD}}^{2}\right)^{2}\right]} \mathcal{F}(s)$.

The first term models the infrared behavior, and the second term is perturbative QCD result [29,31]. It improves the infrared part of Maris-Tandy model to be in concert with modern gauge sector study, while in hadron study the two are equally good. The RL truncation further enforces a ladder truncation to the BS scattering kernel, then the DS-BSEs become solvable [21].

The LF-LFWFs can then be calculated with Eq. (6) using the solved RL $S(k)$ and $\Gamma_{\mu}^{M}(k, P)$. Generally speaking, our method is to calculate the $(2 x-1)$-moments of $\psi_{(i)}^{\Lambda}\left(x, \boldsymbol{k}_{T}^{2}\right)$ at every $\left|\boldsymbol{k}_{T}\right|$, i.e.,

$$
\left\langle(2 x-1)^{m}\right\rangle_{\left|\boldsymbol{k}_{T}\right|}^{(i)}=\int d x(2 x-1)^{m} \psi_{(i)}^{\Lambda}\left(x, \boldsymbol{k}_{T}^{2}\right),
$$

with $m=0,1,2, \ldots$. From these moments we reconstruct the LFWFs. For practical reasons, we treat the $\rho$ and $J / \psi$ with somewhat different techniques.

In solving the $\rho$ DS-BSEs, we only take the infrared part of the QC model, i.e., the first term on the right hand side of Eq. (8). We refer to it as QC-IR model. Since the support of light quark propagator and BS amplitude are dominated by low relative momentum, the ultraviolet term of QC model has 
relatively small effect. Such treatment was also employed in other light quark sector studies [32,33]. Adopting the welldetermined parameters $\omega=0.5 \mathrm{GeV}, D=(0.82 \mathrm{GeV})^{3} / \omega$ $[26,31,34]$ and the current quark mass $m_{u / d}=5 \mathrm{MeV}$, we reproduce $m_{\pi}=131 \mathrm{MeV}$ and $f_{\pi}=90 \mathrm{MeV}$, as well as $m_{\rho}=717 \mathrm{MeV}$ and $f_{\rho}=140 \mathrm{MeV}$ comparing to experimental values $m_{\rho}=775 \mathrm{MeV}$ and $f_{\rho}=156 \mathrm{MeV}$ [35]. We choose QC-IR model rather than QC model as it renders an exponentially $k^{2}-$ suppressed $\Gamma_{\mu}^{M}(k, P)$. This allows us to directly compute up to ninth-moment with Eq. (9), with the numerical noises heavily suppressed. Note with QC model, only the first two or three moments can be directly computed for now. We then fit the moments with a flexible parametrization $[34,36]$

$$
\begin{aligned}
\psi_{(i)}^{\Lambda}\left(x, \boldsymbol{k}_{T}^{2}\right) \approx & {[x(1-x)]^{\alpha-1 / 2} \sum_{j=0,2} a_{j}^{\alpha} C_{j}^{\alpha}(2 x-1) } \\
& +[x(1-x)]^{\alpha^{\prime}-1 / 2} \sum_{j^{\prime}=1,3} a_{j^{\prime}}^{\alpha^{\prime}} C_{j^{\prime}}^{\alpha^{\prime}}(2 x-1),
\end{aligned}
$$

where the $\alpha, \alpha^{\prime}, a_{j}^{\alpha}$, and $a_{j^{\prime}}^{\alpha^{\prime}}$ are fitting parameters. They implicitly depend on the $\boldsymbol{k}_{T}^{2}, \Lambda$ and $i$. The $C_{j}^{\alpha}(x)$ is the Gegenbaur polynomial of order $\alpha$, so the first term on the right-hand side of Eq. (10) is symmetric in $x$ with respect to $x=1 / 2$, and the second term is antisymmetric. They are devised to fit the even and odd (2x-1)-moments separately. Using Eq. (10), we well reproduce all the moments with deviations less than $1 \%$.

For $J / \psi$, we choose the parameters $\omega=0.7 \mathrm{GeV}$ and $D=0.64 \mathrm{GeV}^{2}$ from a recent global analysis on heavy meson spectrum involving both charm and bottom quarks [37]. They are a bit different from that in the light sector, as the DCSB dressing effects they mimic are quantitatively different between light and heavy sectors. In principle, this deviation can be reduced by going beyond RL truncation. Meanwhile we keep the ultraviolet term of the QC model as it is more relevant for heavy quarks. With running quark mass $m_{c}\left(\mu=m_{c}\right)=1.33 \mathrm{GeV}$, we get $m_{J / \psi}=3.09 \mathrm{GeV}$ and $f_{J / \psi}=300 \mathrm{MeV}$, as compared to PDG data $m_{c}\left(\mu=m_{c}\right)=1.28 \mathrm{GeV}, m_{J / \psi}=3.096 \mathrm{GeV}$ and $f_{J / \psi}=$ $294 \mathrm{MeV}$ by leptonic decay $\Gamma\left(J / \psi \rightarrow e^{+} e^{-}\right)=5.53 \mathrm{keV}$

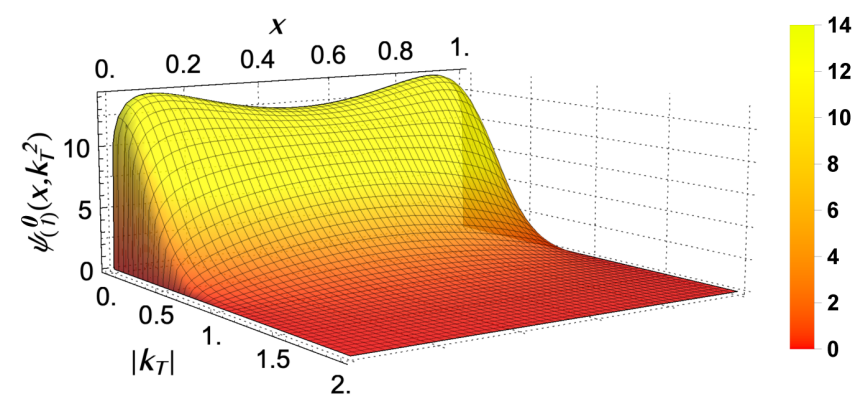

[35]. To compute the $J / \psi$ LFWFs, we adopt the technique used in Refs. [17,38]: by fitting the meson BS amplitude with Nakanishi-like representation [39] and the quark propagator with pairs of complex conjugate poles form which is particularly accurate in heavy sector [40], we are able to compute point-wisely accurate LFWFs. More details can be found in the Supplemental Material [41].

We then obtain all LF-LFWFs of $\rho$ and $J / \psi$. The threedimensional plot of $\psi_{(1)}^{0}\left(x, \boldsymbol{k}_{T}^{2}\right)$ is displayed in Fig. 1 and the rest can be found in the Supplemental Material [41]. They satisfy all the general requirements of Eqs. (2)-(5). Noticeably, the $\rho$ and $J / \psi$ LFWFs are very different in profile. At small and moderate $\boldsymbol{k}_{T}^{2}$, the $J / \psi$ LFWFs are distributed closer to $x=1 / 2$, while the $\rho$ LFWFs are more broadly distributed. This is consistent with the phenomenological $\rho$ LFWFs fitted to diffractive $\rho$ production HERA data [8] and the AdS/QCD prediction [9]. The broadness extends to the twist-2 distribution amplitude (DA) $\phi_{\|}^{V}(x ; \mu)$, which is the $\boldsymbol{k}_{T}$ - integrated LFWF $\phi_{\|}^{V}(x ; \mu)=\frac{\sqrt{6}}{f_{V}} \int^{\left|\boldsymbol{k}_{T}\right|=\mu} \frac{d^{2} \boldsymbol{k}_{T}}{(2 \pi)^{3}} \psi_{(1)}^{0}\left(x, \boldsymbol{k}_{T}^{2}\right)$. For the DA moment $\left\langle\xi^{2}\right\rangle=\left\langle(2 x-1)^{2}\right\rangle$, we obtain $\left\langle\xi^{2}\right\rangle^{\rho}=0.269$ as compared to sum rule results $0.251(24)$ [42], 0.216(21) [43] and $0.241(28)$ [44] at the scale of about $1 \mathrm{GeV}$. Note that we determine our scale for $\rho$ to be $\mu \approx 2 \omega=1 \mathrm{GeV}$, as it is an implicit cutoff within QC-IR model. Meanwhile the lattice QCD gives $\left\langle\xi^{2}\right\rangle^{\rho}=0.268(54)$ [45] and 0.245(9) [46] at a higher scale of $2 \mathrm{GeV}$. For $J / \psi$, we obtain $\left\langle\xi^{2}\right\rangle^{J / \psi}=0.093$ as compared to sum rule results 0.083(12) [47] and $0.070(7)$ [48] and light-front holography prediction $0.096(20)$ [49] at the scale of $\mu=m_{c}$, indicating a significantly narrower DA.

Another difference between the $\rho$ and $J / \psi$ LF-LFWFs is their contribution to Fock-states normalization. The meson's LFWFs of all Fock-states should normalize to unity in general, i.e.,

$$
\begin{aligned}
1 & =\sum_{\lambda, \lambda^{\prime}} N_{\lambda, \lambda^{\prime}}^{\Lambda}+\mathrm{HF}, \\
N_{\lambda, \lambda^{\prime}}^{\Lambda} & =\int_{0}^{1} d x \int \frac{d \boldsymbol{k}_{T}^{2}}{2(2 \pi)^{3}}\left|\Phi_{\lambda, \lambda^{\prime}}^{\Lambda}\left(x, \boldsymbol{k}_{\boldsymbol{T}}\right)\right|^{2} .
\end{aligned}
$$

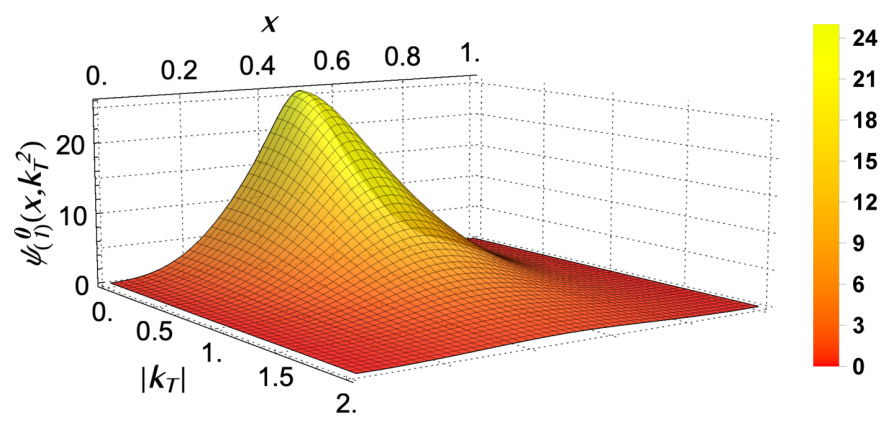

FIG. 1. The LF-LFWF $\psi_{(1)}^{0}\left(x, \boldsymbol{k}_{T}^{2}\right)$ of $\rho$ (left plot) and $J / \psi$ (right plot) respectively. See Eq. (2) for definition of $\psi_{(1)}^{0}\left(x, \boldsymbol{k}_{T}^{2}\right)$. 
TABLE I. LFWFs contribution to Fock-states normalization. See Eq. (12) for definition of $N$.

\begin{tabular}{lccccc}
\hline \hline & $N_{\uparrow, \downarrow}$ & $N_{\downarrow, \uparrow}$ & $N_{\uparrow, \uparrow}$ & $N_{\downarrow, \downarrow}$ & $N_{H F}$ \\
\hline$\rho(\Lambda=0)$ & 0.19 & 0.19 & 0.04 & 0.04 & 0.54 \\
$(\Lambda=1)$ & 0.04 & 0.04 & 0.24 & 0.02 & 0.66 \\
$J / \psi(\Lambda=0)$ & 0.44 & 0.44 & 0.01 & 0.01 & 0.10 \\
$(\Lambda=1)$ & 0.03 & 0.03 & 0.78 & $\approx 0.0$ & 0.16 \\
\hline \hline
\end{tabular}

The HF refers to higher Fock-states. Our result is listed in Table I. The $N_{H F}$ is obtained by subtracting unity with the leading Fock-state contribution. As the DS-BSEs incorporate many Fock-states by summing up infinitely many Feynman diagrams, one can see the higher Fock-states contribute considerably to $\rho$ as compared to $J / \psi$.

\section{DIFFRACTIVE $\rho$ AND $J / \psi$ ELECTROPRODUCTION}

Finally we study the diffractive $\rho$ and $J / \psi$ production $\gamma^{*} p \rightarrow V p$ with the DS-BSEs based LFWFs. In the dipole picture, the process takes three steps: the virtual photon first splits into a color dipole (quark-anti-quark pair), which then scatters off nucleon via color neutral gluons exchange and finally recombines into the outgoing vector meson, leaving the target nucleon intact [50,51]. The scattering amplitude contains (i) the overlap of virtual photon's and vector meson's LF-LFWFs and (ii) the amplitude of dipole scattering off a nucleon. Its revised formula can be found in Refs. [5,52].

We employ the leading order QED result of photon LF-LFWFs [53]

$$
\begin{aligned}
\psi_{\lambda \bar{\lambda}, \Lambda=0}\left(Q^{2}\right)= & -e_{f} e \sqrt{N_{c}} \delta_{\lambda,-\bar{\lambda}} 2 Q x(1-x) \frac{K_{0}(\epsilon r)}{2 \pi} \\
\psi_{\lambda \bar{\lambda}, \Lambda= \pm}\left(Q^{2}\right)= & e_{f} e \sqrt{2 N_{c}}\left\{m_{f} \delta_{\lambda, \pm} \delta_{\bar{\lambda}, \pm}\right. \\
& \left.\left.+i e^{ \pm i \theta_{r}[\mp} x \delta_{\lambda, \pm} \delta_{\bar{\lambda}, \mp} \pm(1-x) \delta_{\lambda, \mp} \delta_{\bar{\lambda}_{, \pm}}\right] \partial_{r}\right\} \\
& \times \frac{K_{0}(\epsilon r)}{2 \pi}
\end{aligned}
$$

with the photon virtuality $Q^{2}$, the quark mass $m_{f}$ and $\epsilon=\sqrt{x(1-x) Q^{2}+m_{f}^{2}}$. Their form in the $\boldsymbol{k}_{T}$-space can be obtained by Fourier transform with respect to the transverse separation $\boldsymbol{r}=\left(r \cos \theta_{r}, r \sin \theta_{r}\right)$. They were originally derived within light cone perturbation theory, with loop corrections available in Refs. [54,55]. Here we remark that Eqs. (13), (14) can also be derived using our method, i.e., calculating Eq. (6) with the bare quark propagator and quark-photon vertex. Naturally, they can be refined by employing the full quark propagator $S$ and vertex $\Gamma_{\mu}$, as the solved $S$ and $\Gamma_{\mu}$ of RL DS-BSEs exhibit considerable dressing effect [56]. In another word, the photon splitting into light $q \bar{q}$ pair contains not only QED, but also essentially nonperturbative QCD interactions. Such study is ongoing within our effort. Nevertheless, at large $Q^{2}$ and/or $m_{f}$ the DCSB effect weakens and the dressed propagator and vertex tend to bare ones. Therefore Eqs. (13), (14) provide a better approximation in the heavy sector, or in the light sector with relatively high $Q^{2}$. Meanwhile, Eqs. (13), (14) inspired some vector meson LFWFs models, such as the Boosted Gaussian model and Gaus-LC model [7,51,57-61]. Their photon-like parametrization satisfies Eqs. (2)-(5), but can not fully accommodate the DS-BSEs LFWFs as we checked.

As for the dipole-proton scattering amplitude, there were many successful models [51,62-64]. Here we adopt the bCGC model [51,64] with parameters determined in Ref. [65]. Note that in analyzing the updated combined HERA small- $x$ DIS data, the bCGC model favors the current light quark mass $m_{u / d}=\left[10^{-4}, 10^{-2}\right] \mathrm{GeV}$ [65]. Here we choose the parameter set with $m_{c}=1.27 \mathrm{GeV}$ and sets $m_{u / d}=5 \mathrm{MeV}$ [65]. We note that another parameter set with $m_{c}=1.4 \mathrm{GeV}$ won't bring much difference to our results.

In Fig. 2 we show the $\gamma^{*} p$ center-of-mass energy $(W)$ dependence of the total cross section $\sigma$ for fixed $Q^{2}$.
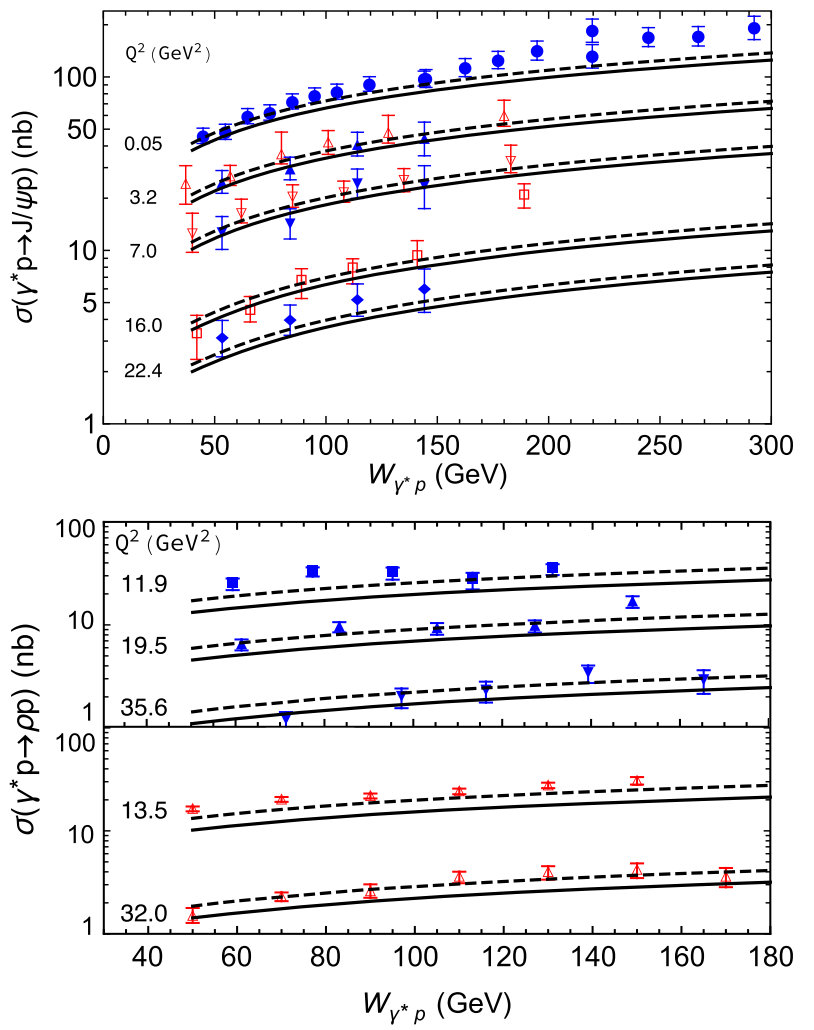

FIG. 2. Upper panel: diffractive $J / \psi$ electroproduction cross section obtained using bCGC model and DS-BSEs LF-LFWFs (solid curves). The dashed curves are 1.1 times the solid curves. The data is taken from H1 [67] (filled markers) and ZEUS [68] (empty markers). Note the selected ZEUS data is at $Q^{2}=$ $3.1 \mathrm{GeV}^{2}$ and $6.8 \mathrm{GeV}^{2}$. Lower panel: results for $\rho$ (solid curves). The dashed curves are 1.3 times solid curves. The data is taken from H1 [69] (filled markers) and ZEUS [70] (empty markers). Deviation grows when $Q^{2}$ gets lower than $10 \mathrm{GeV}^{2}$. 

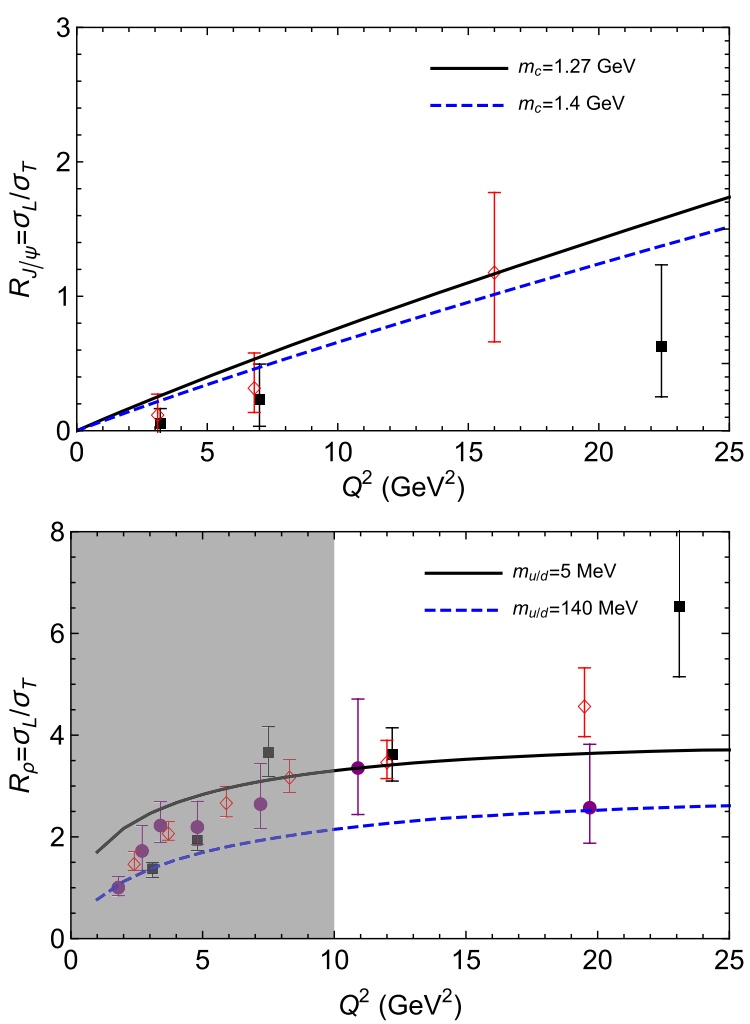

FIG. 3. Longitudinal to transverse cross section ratio of $J / \psi$ and $\rho$ production at $W=90 \mathrm{GeV}$. Upper panel: $J / \psi$ data taken from H1 [67] (filled markers) and ZEUS [68] (empty markers). Lower panel: $\rho$ data taken from H1 $[69,74]$ and ZEUS [70]. The low $Q^{2}$ region is shaded to indicate where the calculation gets less applicable. Different quark mass parameters are examined in the bCGC dipole model.

The upper panel shows our result for $J / \psi$ (solid curves). They generally lie within error bars. As pointed out in Refs. [5,66], there could be an up to $50 \%$ theoretical uncertainty in the overall normalization of the cross section, which originates from the real to imaginary part of the scattering amplitude ratio correction and in particular the skewedness correction. We therefore multiply all the solid curves by a factor of 1.1 and get the dashed curves which show better overall agreement.

The $\rho$ production poses a greater challenge. Since the DS-BSEs LF-LFWFs only contribute less than $50 \%$ to the total normalization, they are significantly smaller in magnitude as compared to phenomenological wave functions that omit higher Fock-states in $\rho$. Meanwhile as aforementioned, there is larger uncertainty in the virtual photon LF-LFWFs concerning $\gamma^{*} \rightarrow q \bar{q}$ as compared to $\gamma^{*} \rightarrow c \bar{c}$ due to nonperturbative effects at low $Q^{2}$ [71-73]. In practice, we find agreement with HERA data for $Q^{2} \gtrsim 10 \mathrm{GeV}^{2}$, as shown in the lower panel of Fig. 2 . The deviation from data shows up as $Q^{2}$ gets lower to around $10 \mathrm{GeV}^{2}$ and keeps growing. For instance at $Q^{2}=3.3 \mathrm{GeV}^{2}$, the data points are about twice our calculation result.

The longitudinal to transverse cross section ratio does not suffer from the absolute normalization uncertainty. We compare HERA data with our calculation in Fig. 3. The quark mass dependence is also examined. Agreement is found in the case of $J / \psi$. We also find the solid curve with $m_{f}=5 \mathrm{MeV}$ goes through more data points than the dashed curve with $m_{f}=140 \mathrm{MeV}$ for $Q^{2} \gtrsim 10 \mathrm{GeV}^{2}$. A definitive conclusion on the preference of $m_{f}$ calls for more data with higher precision in the future.

\section{SUMMARY AND OUTLOOK}

We determine the $\rho$ and $J / \psi$ LF-LFWFs by means of the DS-BSEs approach. Employing the color dipole approach and without introducing any new parameters, these LFWFs well reproduce the diffractive $\rho$ and $J / \psi$ electroproduction data at HERA. This study can be naturally extended to the eA collisions at future EIC. Simulations (within dipole approach) [3] suggest that (i) the diffractive vector meson electroproductions in ep and eA collisions provide good observables for discriminating between saturation and nonsaturation phenomenon and (ii) the lighter vector mesons, such as $\rho$ and $\phi$, are more sensitive probes for gluon saturation. This work paves a new path for obtaining the LF-LFWFs of light vector mesons and hence helpful for their diffractive production study at EIC (and potentially LHeC [75] and EicC [76]) in the future.

\section{ACKNOWLEDGMENTS}

We thank Tobias Frederico, Wen-Bao Jia, Cédric Mezrag, Craig D. Roberts, Peter C. Tandy and Fan Wang for beneficial communications. C.S. also thanks Ian C. Cloët for the help in initiating this project. This work is supported by the National Natural Science Foundation of China (under Grant No. 11905104) and the Strategic Priority Research Program of Chinese Academy of Sciences (Grant No. XDB34030301). 
[1] M. Ryskin, Z. Phys. C 57, 89 (1993).

[2] N. Armesto and A. H. Rezaeian, Phys. Rev. D 90, 054003 (2014).

[3] A. Accardi et al., Eur. Phys. J. A 52, 268 (2016).

[4] S. J. Brodsky, L. Frankfurt, J. Gunion, A. H. Mueller, and M. Strikman, Phys. Rev. D 50, 3134 (1994).

[5] T. Lappi, H. Mäntysaari, and J. Penttala, Phys. Rev. D 102, 054020 (2020).

[6] S. J. Brodsky, H.-C. Pauli, and S. S. Pinsky, Phys. Rep. 301, 299 (1998).

[7] H. Kowalski and D. Teaney, Phys. Rev. D 68, 114005 (2003).

[8] J. Forshaw and R. Sandapen, J. High Energy Phys. 11 (2010) 037.

[9] J. Forshaw and R. Sandapen, Phys. Rev. Lett. 109, 081601 (2012).

[10] M. Ahmady, R. Sandapen, and N. Sharma, Phys. Rev. D 94, 074018 (2016).

[11] G. 't Hooft, Nucl. Phys. B75, 461 (1974).

[12] H. H. Liu and D. E. Soper, Phys. Rev. D 48, 1841 (1993).

[13] M. Burkardt, X.-d. Ji, and F. Yuan, Phys. Lett. B 545, 345 (2002).

[14] C. D. Roberts and A. G. Williams, Prog. Part. Nucl. Phys. 33, 477 (1994).

[15] A. Bashir, L. Chang, I. C. Cloet, B. El-Bennich, Y.-X. Liu, C. D. Roberts, and P. C. Tandy, Commun. Theor. Phys. 58, 79 (2012).

[16] C. Mezrag, H. Moutarde, and J. Rodriguez-Quintero, FewBody Syst. 57, 729 (2016).

[17] C. Shi and I. C. Cloët, Phys. Rev. Lett. 122, 082301 (2019).

[18] W. de Paula, E. Ydrefors, J. H. Alvarenga Nogueira, T. Frederico, and G. Salmè, Phys. Rev. D 103, 014002 (2021).

[19] X.-d. Ji, J.-P. Ma, and F. Yuan, Phys. Rev. Lett. 90, 241601 (2003).

[20] G. P. Lepage and S. J. Brodsky, Phys. Rev. D 22, 2157 (1980).

[21] P. Maris and P. C. Tandy, Phys. Rev. C 60, 055214 (1999).

[22] P. Maris, C. D. Roberts, and P. C. Tandy, Phys. Lett. B 420, 267 (1998).

[23] P. Maris and P. C. Tandy, Phys. Rev. C 62, 055204 (2000).

[24] D. Jarecke, P. Maris, and P. C. Tandy, Phys. Rev. C 67, 035202 (2003).

[25] M. Bhagwat and P. Maris, Phys. Rev. C 77, 025203 (2008).

[26] Y.-Z. Xu, D. Binosi, Z.-F. Cui, B.-L. Li, C. D. Roberts, S.-S. $\mathrm{Xu}$, and H. S. Zong, Phys. Rev. D 100, 114038 (2019).

[27] G. Eichmann, R. Alkofer, A. Krassnigg, and D. Nicmorus, Phys. Rev. Lett. 104, 201601 (2010).

[28] G. Eichmann, Phys. Rev. D 84, 014014 (2011).

[29] P. Maris and C. D. Roberts, Phys. Rev. C 56, 3369 (1997).

[30] J. C. Bloch, Phys. Rev. D 66, 034032 (2002).

[31] S.-x. Qin, L. Chang, Y.-x. Liu, C. D. Roberts, and D. J. Wilson, Phys. Rev. C 85, 035202 (2012).

[32] C. S. Fischer and R. Williams, Phys. Rev. Lett. 103, 122001 (2009).

[33] L. Chang and C. D. Roberts, Phys. Rev. Lett. 103, 081601 (2009).

[34] C. Shi, L. Chang, C. D. Roberts, S. M. Schmidt, P. C. Tandy, and H.-S. Zong, Phys. Lett. B 738, 512 (2014).

[35] P. Zyla et al. (Particle Data Group), Prog. Theor. Exp. Phys. 2020, 083C01 (2020).
[36] C. Shi, C. Chen, L. Chang, C. D. Roberts, S. M. Schmidt, and H.-S. Zong, Phys. Rev. D 92, 014035 (2015).

[37] M. Chen, L. Chang, and Y.-x. Liu, Phys. Rev. D 101, 056002 (2020).

[38] C. Shi, K. Bednar, I. C. Cloët, and A. Freese, Phys. Rev. D 101, 074014 (2020).

[39] N. Nakanishi, Phys. Rev. 130, 1230 (1963).

[40] N. Souchlas, J. Phys. G 37, 115001 (2010).

[41] See Supplemental Material at http://link.aps.org/ supplemental/10.1103/PhysRevD.104.L091902 for details.

[42] P. Ball, V. Braun, and A. Lenz, J. High Energy Phys. 08 (2007) 090.

[43] A. Pimikov, S. Mikhailov, and N. Stefanis, Few-Body Syst. 55, 401 (2014).

[44] H.-B. Fu, X.-G. Wu, W. Cheng, and T. Zhong, Phys. Rev. D 94, 074004 (2016).

[45] R. Arthur, P. Boyle, D. Brommel, M. Donnellan, J. Flynn, A. Juttner, T. Rae, and C. Sachrajda, Phys. Rev. D 83, 074505 (2011).

[46] V. M. Braun et al., J. High Energy Phys. 04 (2017) 082.

[47] H.-B. Fu, L. Zeng, W. Cheng, X.-G. Wu, and T. Zhong, Phys. Rev. D 97, 074025 (2018).

[48] V. Braguta, Phys. Rev. D 75, 094016 (2007).

[49] Y. Li, P. Maris, and J. P. Vary, Phys. Rev. D 96, 016022 (2017).

[50] A. D. Martin, M. Ryskin, and T. Teubner, Phys. Rev. D 62, 014022 (2000).

[51] H. Kowalski, L. Motyka, and G. Watt, Phys. Rev. D 74, 074016 (2006).

[52] Y. Hatta, B.-W. Xiao, and F. Yuan, Phys. Rev. D 95, 114026 (2017).

[53] H. G. Dosch, T. Gousset, G. Kulzinger, and H. Pirner, Phys. Rev. D 55, 2602 (1997).

[54] G. Beuf, Phys. Rev. D 94, 054016 (2016).

[55] H. Hänninen, T. Lappi, and R. Paatelainen, Ann. Phys. (Amsterdam) 393, 358 (2018).

[56] P. Maris and P.C. Tandy, Phys. Rev. C 61, 045202 (2000).

[57] T. Lappi and H. Mantysaari, Phys. Rev. C 83, 065202 (2011).

[58] A. H. Rezaeian, M. Siddikov, M. Van de Klundert, and R. Venugopalan, Phys. Rev. D 87, 034002 (2013).

[59] Y.-p. Xie and X. Chen, Eur. Phys. J. C 76, 316 (2016).

[60] Y.-P. Xie and X. Chen, Nucl. Phys. A959, 56 (2017).

[61] Y.-P. Xie and X. Chen, Nucl. Phys. A970, 316 (2018).

[62] K. J. Golec-Biernat and M. Wusthoff, Phys. Rev. D 59, 014017 (1998).

[63] J. R. Forshaw, G. Kerley, and G. Shaw, Phys. Rev. D 60, 074012 (1999).

[64] E. Iancu, K. Itakura, and S. Munier, Phys. Lett. B 590, 199 (2004).

[65] A. H. Rezaeian and I. Schmidt, Phys. Rev. D 88, 074016 (2013).

[66] D. Boer et al., arXiv:1108.1713.

[67] A. Aktas et al. (H1 Collaboration), Eur. Phys. J. C 46, 585 (2006).

[68] S. Chekanov et al. (ZEUS Collaboration), Nucl. Phys. B695, 3 (2004). 
[69] F. Aaron et al. (H1 Collaboration), J. High Energy Phys. 05 (2010) 032.

[70] S. Chekanov et al. (ZEUS Collaboration), PMC Phys. A 1, 6 (2007).

[71] J. R. Forshaw, R. Sandapen, and G. Shaw, Phys. Rev. D 69, 094013 (2004).

[72] J. Berger and A. M. Stasto, J. High Energy Phys. 01 (2013) 001.
[73] V. P. Gonçalves and B. D. Moreira, Eur. Phys. J. C 80, 492 (2020)

[74] C. Adloff et al. (H1 Collaboration), Eur. Phys. J. C 13, 371 (2000).

[75] P. Agostini et al. (LHeC, FCC-he Study Group), arXiv: 2007.14491.

[76] D. P. Anderle et al., Front. Phys. (Beijing) 16, 64701 (2021). 